\title{
A High-Efficient CMOS RF Power Amplifier With Automatic Adaptive Bias Control
}

\author{
Yi-Jan Emery Chen, Member, IEEE, Chih-Yun Liu, Tang-Nian Luo, and Deukhyoun Heo
}

\begin{abstract}
A 2.4-GHz Doherty power amplifier (PA) is developed in 0.18- $\mu \mathrm{m}$ CMOS technology. An automatic adaptive bias control circuit is integrated with the auxiliary PA to improve the overall performance of the PA. Operated at $3 \mathrm{~V}$, the $P_{1} \mathrm{~dB}$ and associated power-added-efficiency (PAE) of the $P A$ are $21 \mathrm{dBm}$ and $33 \%$, respectively. At the output power 6-dB backoff from $P_{1 \mathrm{~dB}}$, the PAE remains $21 \%$. The limited $P A E$ degradation at backoff power levels makes the PA suitable for the applications with high peak-to-average power ratio.
\end{abstract}

Index Terms-Adaptive bias, CMOS, Doherty power amplifier (PA), orthorgonal frequency division multiplexing (OFDM), peak-to-average power ratio (PAPR).

\section{INTRODUCTION}

$\mathbf{O}$ RTHORGONAL frequency division multiplexing (OFDM) is a very popular modulation technique for modern high-speed wireless communications because of robustness against multipath fading [1]. However, in the context of OFDM, specific data sequences will lead to a large peak-to-average power ratio (PAPR) of the output signal [2]. The characteristics of high PAPR is a significant issue for the traditional radio frequency (RF) power amplifier (PA) because the efficiency at the average output power level will degrade dramatically from that at the saturation power level. The Doherty PA architecture is a promising solution to the high PAPR issue of the OFDM signals since it can maintain high efficiency at the backoff output power [3], [4].

This letter presents a 2.4-GHz Doherty PA developed in a commercial $0.18-\mu \mathrm{m}$ dual-oxide CMOS technology. Because the CMOS devices have less driving capability than the III-V or SiGe transistors, the intuitive bias scheme for the auxiliary amplifier is incapable of delivering enough power or achieving decent efficiency [5]. The adaptive biasing is one of the techniques effective for improving the efficiency of RF PAs [6]. A novel adaptive bias control circuit is proposed for the auxiliary PA biasing to enhance the efficiency and output power characteristics of the Doherty PA. Operated on a 3-V supply, the

Manuscript received April 11, 2006; revised June 22, 2006. This work was supported in part by the Taiwan National Science Council of Taiwan, R.O.C., under Grant 92-2218-E-002-057, the National Chip Implementation Center, and the NTU-MediaTek Wireless Research Laboratory.

Y.-J. E. Chen, C.-Y. Liu, and T.-N. Luo are with the Graduate Institute of Electronics Engineering, Graduate Institute of Communication Engineering, and Department of Electrical Engineering, National Taiwan University, Taipei 106, Taiwan, R.O.C. (e-mail: emery @ cc.ee.ntu.edu.tw).

D. Heo is with the School of Electrical Engineering and Computer Science, Washington State University, Pullman, WA 99164 USA

Color versions of Figs. 1-7 are available online at http://ieeexplore.org.

Digital Object Identifier 10.1109/LMWC.2006.884909

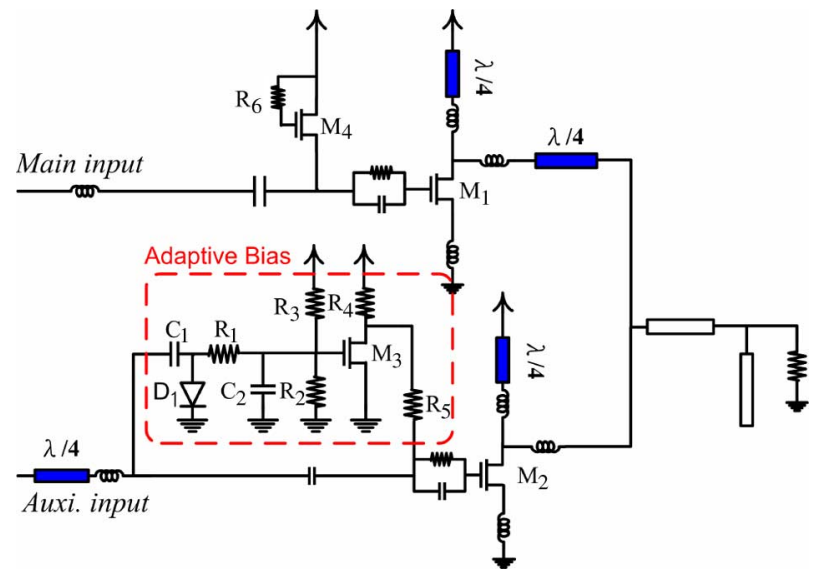

Fig. 1. Simplified schematic diagram of the CMOS PA with the adaptive bias control circuit.

$P_{1 \mathrm{~dB}}$ and associated power-added-efficiency (PAE) of the PA are $21 \mathrm{dBm}$ and $33 \%$, respectively. The PAE at the output power 6-dB backoff from $P_{1 \mathrm{~dB}}$ is $21 \%$. The PAE degradation due to power backoff $6 \mathrm{~dB}$ from $P_{1 \mathrm{~dB}}$ is only $36 \%$.

\section{ADAPTIVE BIAS CONTROL}

The simplified schematic diagram of the CMOS PA and the adaptive bias circuit is shown in Fig. 1. A small fraction of the input power into the auxiliary PA will go through a half-wave rectifier consisting of $C_{1}$ and $D_{1}$. The rectified signal is fed into a low-pass filter $\left(R_{1}-C_{2}\right)$ to obtain a negative dc value. The larger the input power, the lower the negative dc voltage. The values of $R_{1}$ and $C_{2}$ will affect the bandwidth of the bias circuit. The negative dc voltage is superimposed on the gate voltage of the bias control device, $M_{3}$. The $M_{3}$ is initially biased in saturation region as a voltage-controlled current source. The drain voltage of $M_{3}$ is applied to the bias of the auxiliary power device through a large resistor $R_{5}$.

For low input driving levels, the magnitude of the negative dc voltages obtained after the low-pass filtering are small so the drain voltage of $M_{3}$ remains low and the auxiliary power device is not turned on. As the input driving level increases, the negative dc voltage from the low-pass filter will lower the gate voltage of $M_{3}$. The drain current of $M_{3}$ will decrease and the drain voltage of $M_{3}$ increases accordingly. Therefore, the bias voltage of the auxiliary power device will increase with the input power automatically. The turn-on threshold of the auxiliary PA can be controlled by $R_{1}$, as shown in Fig. 2 , or the ratio of $R_{2}$ and $R_{3}$. The slope of the adaptive bias characteristics can be tuned by $R_{4}$, as shown in Fig. 3. By proper selection of $R_{2}, R_{3}$, 


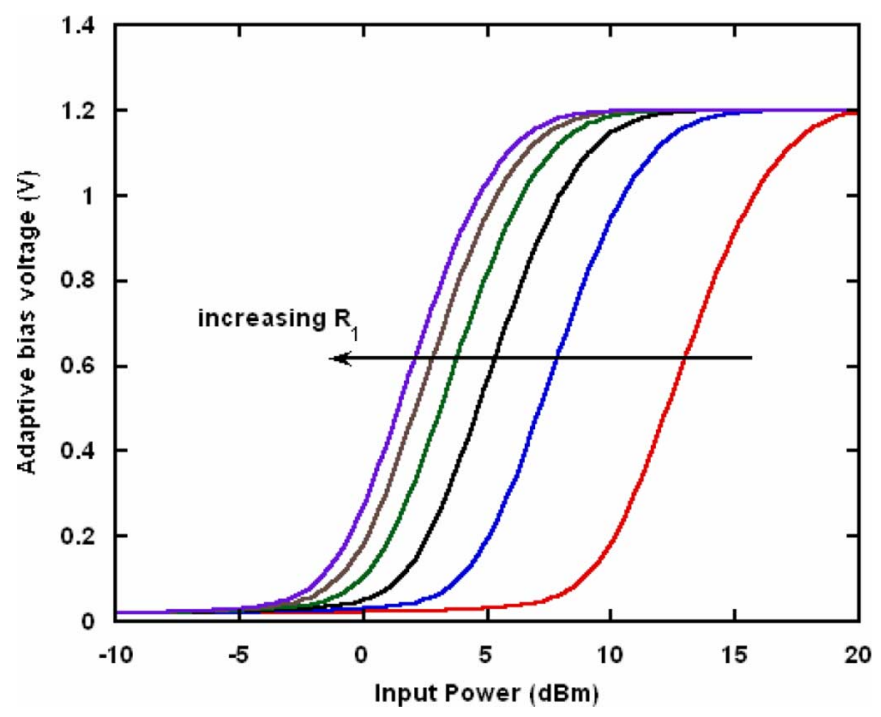

Fig. 2. Characteristics of adaptive bias voltage with respect to input power for $R 1$ varying from 10 to $60 \Omega$.

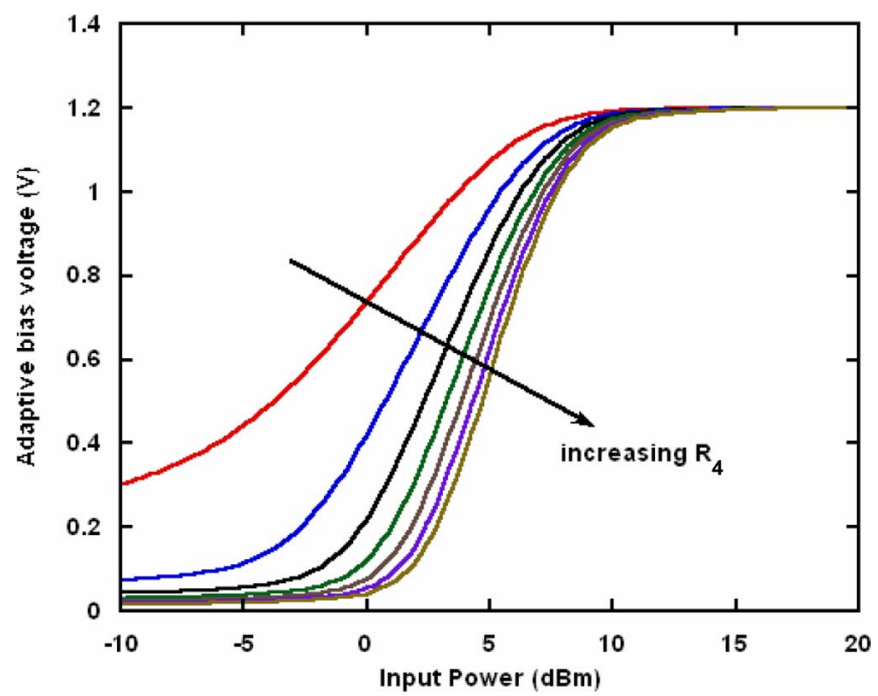

Fig. 3. Characteristics of adaptive bias voltage with respect to input power for $R_{4}$ varying from $1.5 \mathrm{k}$ to $4.5 \mathrm{k} \Omega$.

and $R_{4}$, the desired adaptive bias characteristics can be implemented. The bandwidth of the proposed adaptive bias control can reach $20 \mathrm{MHz}$.

\section{POWER AMPLIFIER IMPLEMENTATION}

The 2.4-GHz Doherty PA chip was designed using UMC $0.18-\mu \mathrm{m}$ CMOS technology. The CMOS technology has the dual gate option and the thick-oxide devices are used only for the PA implementation because of higher breakdown voltage. The total gate widths of the main and auxiliary power devices are $630 \mu \mathrm{m}$ and $840 \mu \mathrm{m}$, respectively. The design goal is to improve the efficiency at the power 6-dB backoff from $P_{1 \mathrm{~dB}}$. Theoretically, the auxiliary power device size should be at least twice as large as the main power device for the 6-dB backoff design goal [4]. Thanks to the adaptive bias control, the auxiliary

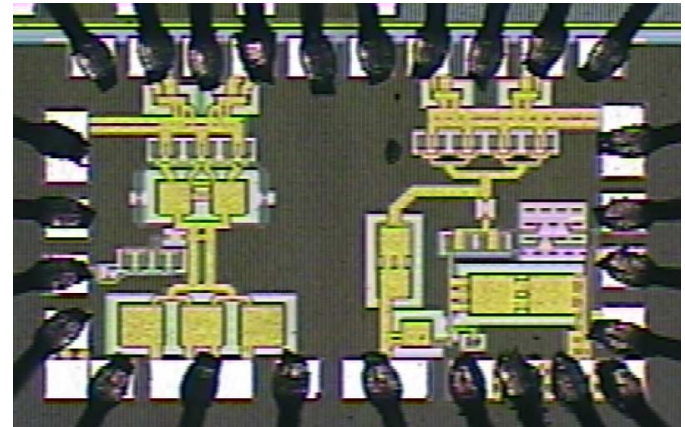

Fig. 4. Microphotograph of the CMOS PA chip. The total chip size is $0.9 \times$ $0.6 \mathrm{~mm}^{2}$.

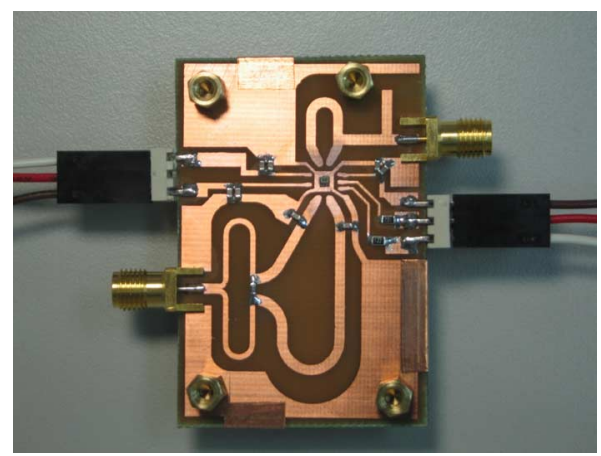

Fig. 5. Photograph of the 2.4-GHz Doherty PA. The CMOS chip is mounted on the two-layer 32-mil-thick FR-4 board.

power device size can be reduced significantly, and the overall efficiency of the PA can be enhanced accordingly. In addition, smaller CMOS power device size implies less parasitics and better achievable power gain. The diode-connected MOSFET is used to bias the main power device, as shown in Fig. 1. In addition to providing proper bias, the diode-connected MOSFET can improve the linearity of the main power device [7]. The main power device of the Doherty amplifier is biased at class $\mathrm{AB}$ to provide linear output power when the auxiliary power device is not turned on. If the main device is biased at class B or $\mathrm{C}$, the power gain flatness will be degraded in the low power operation regime. The auxiliary power device was biased by the adaptive bias circuit. The simulation shows that the proposed adaptive bias circuit results in the characteristics leading to excellent output power and efficiency. The microphotograph of the CMOS chip is shown in Fig. 4 and the total chip size is $0.9 \times 0.6 \mathrm{~mm}^{2}$.

The CMOS PA chip is mounted on a two-layer 32-mil-thick FR-4 board for testing, as shown in Fig. 5. The quarter-length transmission lines and the Wilkinson power divider used in the Doherty PA are implemented on the FR-4 board. The Wilkinson divider exhibits $0.3-\mathrm{dB}$ insertion loss around $2.4 \mathrm{GHz}$. The measured $S$-parameters of the Wilkinson divider are shown in Fig. 6. The two signal paths to the main and auxiliary power devices are carefully designed and compensated to minimize phase difference. The impedance transformation and matching at the output are achieved with the transmission lines and open stub on the FR-4 board. 


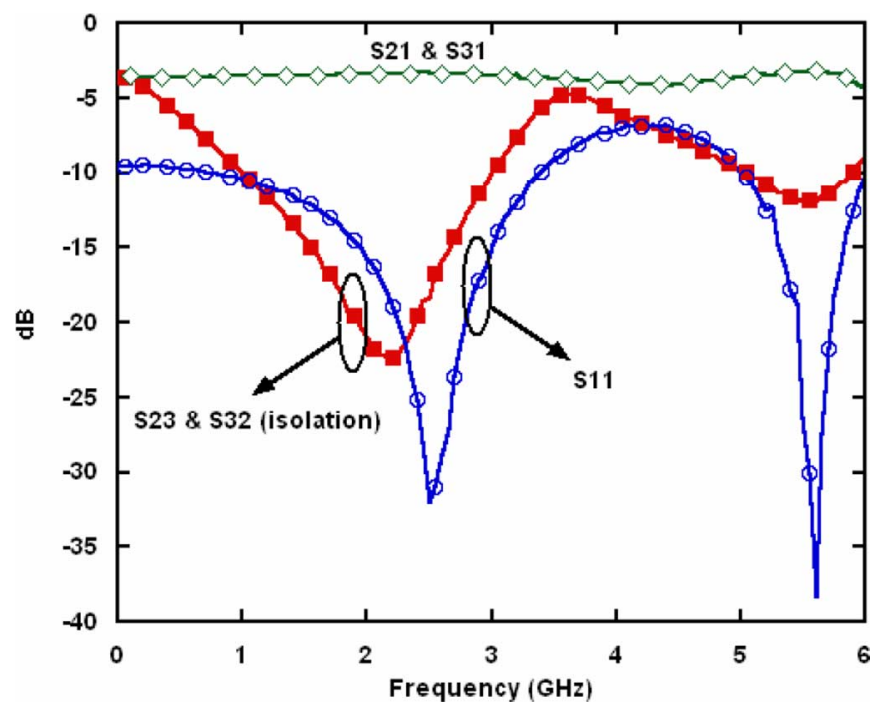

Fig. 6. Measured $S$-parameters of the Wilkinson power divider. Port 1 is the input port and Port 2 and 3 are output ports.

\section{Measurement Results}

The Doherty PA is powered by a $3-\mathrm{V}$ supply. The measured power gain, output power, and PAE with respect to input power are shown in Fig. 7. The power gain of the PA is $10.6 \mathrm{~dB}$, and the maximum output power is $22.3 \mathrm{dBm}$. The $P_{1 \mathrm{~dB}}$ is $21.4 \mathrm{dBm}$ and the corresponding PAE is $33 \%$. The PAE at the power 6-dB backoff from $P_{1 \mathrm{~dB}}$ is $21 \%$. The PAE degradation due to the power backoff is calculated as

$\Upsilon(\alpha \mathrm{dB})=\frac{\left[\text { PAE at } P_{1 \mathrm{~dB}}\right]-\left[\mathrm{PAE} \text { at }\left(P_{1 \mathrm{~dB}}-\alpha\right)\right]}{\text { PAE at } P_{1 \mathrm{~dB}}} \times 100 \%$.

The measured PAE degradation due to 6-dB backoff from $P_{1 \mathrm{~dB}}, \Upsilon(6 \mathrm{~dB})$, of the CMOS Doherty PA is only $36 \%$. To the authors' knowledge, the $6 \mathrm{~dB}$-backoff PAE degradation of this work is best of the CMOS RF PAs published.

\section{CONCLUSION}

A 2.4-GHz Doherty PA is implemented in a commercial $0.18-\mu \mathrm{m}$ CMOS technology. An automatic adaptive bias control circuit is integrated with the auxiliary PA to improve the

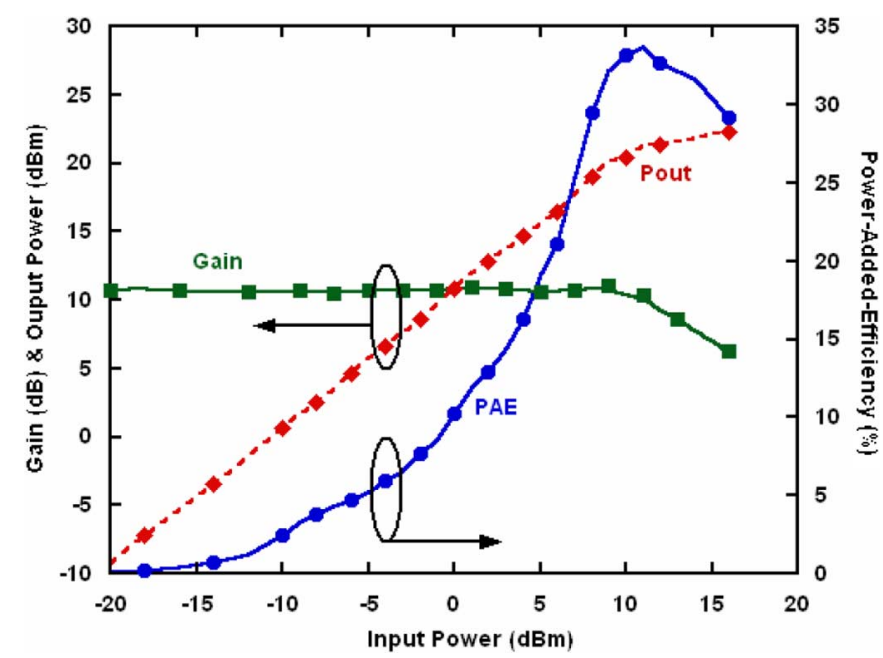

Fig. 7. Measured power gain, output power, and PAE of the CMOS Doherty PA.

performance of the Doherty PA. Operated on a 3-V supply, the CMOS PA achieves the peak power of $22.3 \mathrm{dBm}$. The measured power gain and $P_{1} \mathrm{~dB}$ are $10.6 \mathrm{~dB}$ and $21.4 \mathrm{dBm}$, respectively. The PAE degradation at the power $6-\mathrm{dB}$ backoff from $P_{1 \mathrm{~dB}}$ is as low as $36 \%$.

\section{REFERENCES}

[1] V. Chakravarthy, A. S. Nunez, and J. P. Stephens, "TDCS, OFDM, and MC-CDMA: a brief tutorial," IEEE Commun. Mag., vol. 43, no. 9, pp. S11-S16, Sep. 2005.

[2] H. Ochiai and H. Imai, "On the distribution of the peak-to-average power ratio in OFDM signals," IEEE. Trans. Commun., vol. 49, no. 2, pp. 282-289, Feb. 2001.

[3] W. H. Doherty, "A new high efficiency power amplifier for modulated waves," Proc. IRE, vol. 24, no. 9, pp. 1163-1182, Sep. 1936.

[4] S. C. Cripps, RF Power Amplifiers for Wireless Communications. Norwood, MA: Artech House, 1999.

[5] C.-Y. Liu, Y.-J. E. Chen, and D. Heo, "Impact of bias schemes on Doherty power amplifiers," in Proc. IEEE Int. Symp. Circ. Syst., May 2005, vol. 1, pp. 212-215.

[6] J. Cha, Y. Yang, B. Shin, and B. Kim, "An adaptive bias controlled power amplifier with a load-modulated combining scheme for high efficiency and linearity," in IEEE MTT-S Int. Dig., Jun. 2003, pp. 81-84.

[7] C.-C. Yen and H.-R. Chuang, "A 0.25- $\mu \mathrm{m}$ 20-dBm 2.4-GHz CMOS power amplifier with an integrated diode linearizer," IEEE Microw. Wireless Compon. Lett., vol. 13, no. 2, pp. 45-47, Feb. 2003. 